\title{
Desarrollo de la técnica de disección submucosa endoscópica en cáncer gástrico incipiente. Primeros cincuenta casos de un cirujano
}

\author{
Ricardo Javier Mejía Martínez ${ }^{1}$
}

TRABAJO DE INGRESO A SOCIEDAD

\section{Development of endoscopic submucosal dissection for early gastric cancer. Fifty first cases of a surgeon}

Introduction: Gastric cancer is a serious health problem in Chile and its poor prognosis is explained by usual diagnosis in advanced stages. Early gastric cancer (EGC) is associated with good oncological prognosis and endoscopic submucosal dissection (ESD) as its treatment has shown to be comparable to the surgical standard. Aim: Describe the experience and results of a surgeon in patients with EGC undergoing ESD. Materials and Method: Retrospective study including patients undergoing ESD for EGC between May 2015 and March 2019. Demographic information, perioperative and follow-up data obtained from the institutional registry were tabulated. Descriptive statistics was used. Results: 52 ESD were performed per EGC in the analyzed period. En bloc resection was achieved in $96 \%$ of cases. Morbidity reached $2 \%$ and no postoperative mortality was recorded. $98 \%$ of the en bloc resections were considered R0 and $86 \%$ of them met healing standards. The median follow-up is 19 months and the specific survival due to gastric cancer and recurrence-free rates reach 100\%. Discussion: ESD is a technique with curative potential in patients with EGC, presenting better results in terms of morbidity and mortality, quality of life and is comparable to surgery in the oncological prognosis. The results presented are consistent with the literature and support the implementation of the technique for the treatment of EGC.

Key words: early gastric cancer; submucosal dissection; endoscopic treatment.

\section{Resumen}

Introducción: El cáncer gástrico es un problema de salud en Chile y su mal pronóstico global se explica por un diagnóstico en etapas avanzadas. El cáncer gástrico incipiente (CGI) se asocia a buen pronóstico oncológico y la disección submucosa endoscópica (DSE) como alternativa de tratamiento ha demostrado ser comparable al estándar quirúrgico. Objetivo: Describir la experiencia y resultados de un cirujano en pacientes con CGI sometidos a DSE. Materiales y Método: Estudio retrospectivo que incluyó a los pacientes sometidos a DSE por CGI entre mayo de 2015 y marzo de 2019. Se tabuló información demográfica, datos perioperatorios y del seguimiento obtenidos del registro institucional. Se utilizó estadística descriptiva. Resultados: Se realizaron 52 DSE por CGI en el período analizado. La resección en bloque se logró en un 96\% de los casos. La morbilidad alcanzó el 2\%, no se registró mortalidad postoperatoria. $98 \%$ de las resecciones en bloque fueron consideradas R0 y un $86 \%$ de ellas cumplieron estándares de curación. La mediana de seguimiento es de 19 meses y la sobrevida específica por cáncer gástrico y libre de recurrencia alcanza el 100\%. Discusión: La DSE es una técnica con potencial curativo en pacientes con CGI, presentando mejores resultados en términos de morbimortalidad, calidad de vida y es comparable a la cirugía en el pronóstico oncológico. Los resultados presentados son concordantes con la literatura y apoyan la implementación de la técnica para el tratamiento del CGI.

Palabras clave: cáncer gástrico incipiente; disección submucosa; tratamiento endoscópico.
Institución Pontificia Universidad Católica de Chile. Santiago, Chile.

Recibido el 15 de septiembre de 2019, aceptado para publicación el 27 de septiembre de 2019.

Correspondencia a: Dr. Ricardo Javier Mejía Martínez rjmejiam@gmail.com 


\section{Introducción}

El cáncer gástrico es un grave problema de salud en Chile. Constituye la $1^{\text {a }}$ causa de muerte por tumores malignos en hombres y la tercera en mujeres, dando cuenta de más de 3.000 muertes al año․

El mal pronóstico global de esta enfermedad se debe principalmente a que la gran mayoría de los pacientes es diagnosticado en etapas avanzadas ${ }^{2,3}$. Esto se asocia a malos resultados oncológicos independiente del tratamiento realizado $o^{4,5}$.

El cáncer gástrico incipiente (CGI) se define como aquel que compromete la mucosa y/o la submucosa independiente del compromiso linfático. En contraste a lo que ocurre en etapas avanzadas, la cirugía del cáncer gástrico incipiente se asocia a un excelente pronóstico oncológico a largo plazo, con sobrevidas que alcanzan hasta el $90 \%$ a los 5 años $^{6-10}$.

La gastrectomía con disección ganglionar es considerada como el estándar de tratamiento para estos pacientes, sin embargo se asocia a una morbimortalidad perioperatoria y a largo plazo no despreciable ${ }^{11,12}$.

La disección submucosa endoscópica (DSE) es una técnica quirúrgica que se asocia a tasas de morbimortalidad bajas y que preserva el estómago, teniendo menor impacto sobre la calidad de vida ${ }^{13-16}$. Desde su descripción ha habido un aumento exponencial en el número de procedimientos realizados en todo el mundo y especialmente en países asiáticos, donde se concentra la mayor experiencia en esta técnica. Inicialmente estaba reservada para pacientes con cáncer gástrico bien diferenciado, menor a 2 centímetros y no ulcerado ${ }^{17}$.

Al aumentar la experiencia en la técnica y comprensión de la enfermedad, se ha ampliado su indicación. Es así como en el año 2000 se popularizaron los criterios expandidos de DSE, basado en el estudio de piezas quirúrgicas de pacientes sometidos a gastrectomía con disección ganglionar por CGI, identificando a aquellos grupos de pacientes con nula o muy baja probabilidad de metástasis linfáti$\operatorname{cas}^{17}$ (Tabla 1).

Tabla 1. Criterios expandidos para disección submucosa endoscópica (DSE) en cáncer gástrico incipiente (CGI)

Tumor intramucoso, bien diferenciado, no ulcerado sin límite de tamaño

Tumor intramucoso, bien diferenciado, ulcerado y tamaño menor a $3 \mathrm{~cm}$

Tumor intramucoso, mal diferenciado, no ulcerado y tamaño menor a $2 \mathrm{~cm}$

Tumor bien diferenciado con compromiso submucoso menor a 500 micras y tamaño menor a $3 \mathrm{~cm}$
Aún hoy la DSE en el tratamiento del CGI en pacientes que cumplen con los criterios expandidos es considerada por la Sociedad Japonesa de Cáncer Gástrico como una alternativa terapéutica "en investigación"18; aunque cada vez hay más publicaciones disponibles que la avalan como una alternativa con resultados oncológicos comparables a la gastrectomía con resección ganglionar ${ }^{19,20}$.

Los reportes en centros de occidente y en particular en nuestro país son más escasos ${ }^{21,22}$. El objetivo de este trabajo es describir la experiencia y resultados de un cirujano en pacientes con CGI sometidos a disección submucosa.

\section{Materiales y Método}

Estudio retrospectivo descriptivo de una base de datos registrada de manera prospectiva. Se realizó en el Complejo Asistencial Dr. Sótero del Río, contemplando el período comprendido desde mayo de 2015 a marzo de 2019.

Se incluyeron los pacientes sometidos a DSE por CGI. La indicación fue evaluada por el equipo de cirugía esófago-gástrica, luego de una discusión individualizada y contando con etapificación clínica. Todas las resecciones fueron realizadas por un cirujano digestivo con experiencia en endoscopia terapéutica.

Registramos datos epidemiológicos, perioperatorios y del seguimiento, obtenidos desde el registro institucional.

En la etapa preoperatoria los pacientes fueron evaluados de manera clínica y con hemograma, pruebas de función hepática, renal y electrocardiograma. El diagnóstico fue realizado con endoscopía digestiva alta y biopsia, la etapificación incluyó una tomografía de tórax, abdomen y pelvis con contraste. Sólo se incluyeron pacientes con diagnóstico preoperatorio de adenocarcinoma gástrico o displasia epitelial, otras histologías fueron excluidas.

El procedimiento fue realizado de acuerdo al cumplimiento de los criterios expandidos de disección endoscópica aceptados por la Sociedad Japonesa de Cáncer Gástrico ${ }^{18}$.

\section{Técnica quirúrgica}

Todos los pacientes fueron sometidos a la DSE en pabellón, con anestesia general y relajo muscular. Se utilizó un endoscopio diagnóstico Olympus ${ }^{\circledR}$ serie 180 o 190 con un espaciador distal. La insuflación fue realizada con $\mathrm{CO}_{2}$.

La disección se inició luego de identificar la lesión con el uso de endoscopía de luz blanca y cromoendoscopía digital o con índigo carmín. 
Luego se procedió a la marcación de los bordes con electrocoagulación para continuar con la elevación del espacio submucoso con Voluven ${ }^{\circledR}$, índigo carmín y adrenalina. La mucotomía y posterior disección submucosa se llevaron a cabo con cuchillos endoscópicos procurando una resección en bloque de la pieza (Figura 1). Al terminar el procedimiento se realizó hemostasia con pinzas de coagulación y se verificó la indemnidad de la pared gástrica.

Luego de completar la resección se procedió en todos los casos a extender la pieza fijándola en sus extremos y, posteriormente, enviarla en formalina para su estudio anatomopatológico definitivo. Se evaluó dirigidamente el tipo histológico, tamaño tumoral, grado de diferenciación, presencia de permeaciones linfovasculares, bordes laterales y pro- fundo de las piezas para determinar el rol curativo de dichas resecciones.

Los pacientes permanecieron las primeras horas en el servicio de recuperación y luego trasladados a cama quirúrgica en sala de baja complejidad. Se mantuvieron en régimen cero las primeras $24 \mathrm{~h}$ postoperatorias, con hidratación endovenosa, inhibidor de bomba de protones, antieméticos y analgesia multimodal. Se evitó el uso de anticoagulantes y se favoreció la deambulación precoz.

La dieta líquida se inició al día siguiente y luego papilla en el día subsiguiente, la que se mantuvo hasta el primer control postoperatorio. El alta se definió regularmente a las $48 \mathrm{~h}$ del procedimiento de acuerdo a la tolerancia al régimen y manejo del dolor.

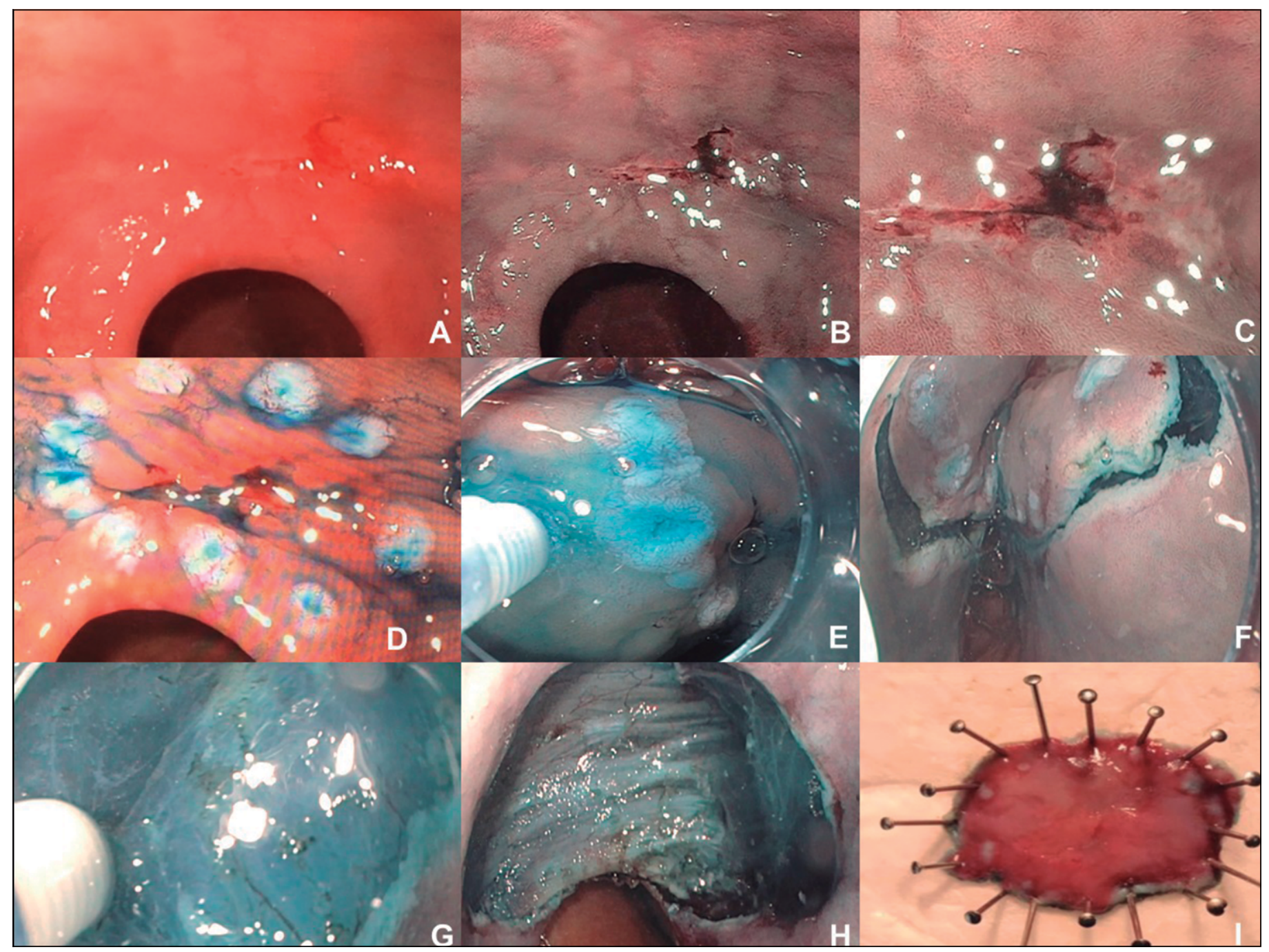

Figura 1. Técnica de DSE. A: Endoscopía de luz blanca. B y C: Cromoendoscopía digital. D: Marcación de la lesión. E: Elevación de la submucosa. F: Mucotomía circunferencial. G: Disección submucosa. H: Lecho de resección. I: Pieza extendida. 


\section{ARTÍCULO ORIGINAL}

\section{Seguimiento}

El seguimiento es realizado mediante control clínico, endoscópico y biopsias realizadas de manera rutinaria a los 3, 6 y 12 meses. Posteriormente, los pacientes se mantienen en control endoscópico anual.

\section{Análisis estadístico}

Fueron analizadas variables demográficas, características clínicas, del diagnóstico endoscópico e histológico preoperatorio, datos del procedimiento y morbilidad asociada, resultado histológico definitivo y seguimiento. Se evaluó la sobrevida global y libre de enfermedad.

Las variables categóricas se expresan en valores numéricos y porcentajes, las variables continuas en medianas y rangos. El análisis estadístico se realizó con SPSS v24, Inc, Chicago, IL.

El presente trabajo fue aprobado por el comité de ética local.

\section{Resultados}

Un total de 52 DSE fueron realizadas en 51 pacientes entre el año 2015 y 2019. El 61,8\% corresponde a pacientes de género femenino con una edad promedio de 65 años (50-87). Un 80\% correspondían a categoría ASA II y $9 \%$ a ASA III.

La ubicación de las lesiones de acuerdo a la división anatómica del estómago en tercios fue la

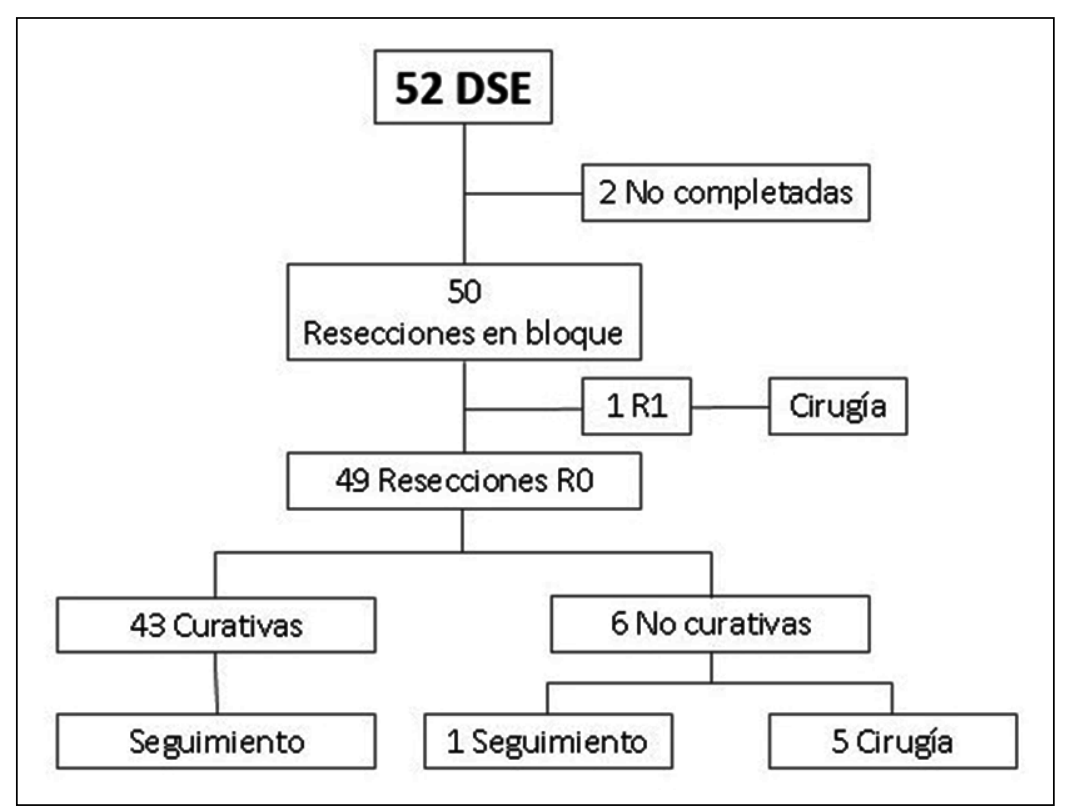

Figura 2. Pacientes sometidos a DSE por CGI. siguiente; tercio superior $35 \%$, tercio medio $30 \%$ y tercio inferior $35 \%$.

La histología preoperatoria incluyó 30 adenocarcinomas, 21 lesiones con displasia de alto grado (DAG) y 1 con displasia de bajo grado.

La resección en bloque fue lograda en el $96 \%$ de los casos. En 2 pacientes (4\%) esto no fue alcanzado; un caso por imposibilidad de elevar la submucosa y un segundo paciente que presentó un shock anafiláctico durante la disección que obligó a suspender el procedimiento (Figura 2).

La tasa de complicaciones fue de un $2 \%$. Un paciente presentó una hemorragia digestiva que fue manejada satisfactoriamente mediante endoscopía (Clavien IIIa). No se registraron perforaciones. No se presentaron casos de mortalidad perioperatoria.

Un paciente presentó una estenosis pilórica durante el seguimiento y presentó mala respuesta a dilataciones endoscópicas repetidas por lo que debió ser sometida a una gastro-yeyuno anastomosis laparoscópica.

La mediana de estadía hospitalaria para los pacientes con resección en bloque fue de 2 días, con un rango entre 2 y 5 días.

Del total de las resecciones en bloque $98 \%$ fueron consideradas R0. En un solo caso se informó borde lateral y profundo positivo, paciente sometido a una gastrectomía total laparoscópica como tratamiento definitivo y cuya biopsia informó un adenocarcinoma residual.

De acuerdo a los criterios expandidos, 43 (86\%) de las disecciones submucosas catalogadas como R0, cumplieron con estándares de curación. De los pacientes restantes, tres fueron sometidos a gastrectomía total laparoscópica y dos a gastrectomía subtotal laparoscópica. En el análisis de estas piezas quirúrgicas, solo en una se demostró compromiso ganglionar. En un paciente se definió seguimiento debido a la baja probabilidad de metástasis ganglionares, edad avanzada y comorbilidades (Figura 2).

A la fecha no se han registrado recurrencias.

La mediana de seguimiento es de 19 meses (143). La sobrevida global de la serie es de un $98 \%$, solo registrándose un caso de mortalidad secundario a una leucemia mieloide aguda. La sobrevida específica por cáncer gástrico es de $100 \%$ y la sobrevida libre de recurrencia de un $100 \%$.

\section{Discusión}

La sobrevida global de pacientes diagnosticados de cáncer gástrico es pobre. Esto debido a que la gran mayoría de ellos, en países no asiáticos, son detectados en estadios avanzados ${ }^{2}$. En contraste a 
esto la sobrevida a 5 años de pacientes con CGI supera el $90 \%{ }^{6}$.

El estándar de tratamiento actual en casos de CGI es la gastrectomía total o subtotal con disección ganglionar $^{18}$. Esto permite el control local del tumor primario y la resección de los linfonodos que pudieran estar comprometidos. Se estima una invasión linfática tumoral en aproximadamente 3\% de los tumores mucosos y hasta $23 \%$ de los tumores sub$\operatorname{mucosos}^{23}$. En nuestra experiencia en gastrectomías con disección ganglionar sólo un $6 \%$ de los tumores T1a o T1b tenían compromiso linfático ${ }^{24}$ con lo cual en el $94 \%$ de estos pacientes podría haberse evitado dicha intervención.

El problema de identificar a aquellos pacientes con nulo riesgo o muy bajo riesgo de metástasis linfáticas fue resuelto mediante el trabajo publicado por Gotoda y cols el año $2000^{17}$. Esto permitió definir a aquellos grupos en los cuales una disección linfática no sería necesaria y sienta las bases de la DSE en la cual se realiza sólo tratamiento del tumor primario.

La DSE constituye una herramienta diagnóstica "macrobiopsia" con potencial curativo en cerca de $90 \%$ de los pacientes. De los aspectos más relevantes a considerar son la menor morbilidad y prácticamente nula mortalidad asociada a la técnica lo que plantea claros beneficios respecto a la cirugía resectiva tradicional. En nuestro hospital, un centro de alto volumen en que se operan aproximadamente 70 gastrectomías al año, hemos reportado una mortalidad en gastrectomías por cáncer gástrico incipiente de $2,6 \% \%^{24}$. Lo anterior permite estimar que 1 a 2 pacientes de nuestra serie hubieran fallecido en el período postoperatorio sólo por el hecho de haber sido sometidos a una gastrectomía, cuando esta podría haberse evitado.

La tasa de complicaciones de la serie actual es comparable a la descrita en grandes series internacionales de $\mathrm{DSE}^{25}$. En la experiencia inicial previamente publicada por nuestro grupo sobre DSE en CGI se presentaron complicaciones en un $26 \%$ (perforación y hemorragia) $^{22}$. Con respecto a ese período, se realizaron modificaciones al abordaje y manejo de estos pacientes: se fijó como estándar en nuestra institución la realización de esta cirugía en pabellón, con anestesia general, relajo muscular, insuflación con $\mathrm{CO}_{2}$ y endoscopios de alta calidad. Lo anterior también fue facilitado por la implementación de la canasta GES para la DSE del CGI. El impacto de las medidas descritas se ha traducido en una reducción de la morbilidad del procedimiento a $2 \%$, lo que se compara favorablemente con lo reportado en complicaciones postoperatorias de gastrectomía.
Mención aparte representa la potencial diferencia en términos de calidad de vida luego de una DSE versus gastrectomía, especialmente en el caso de la gastrectomía total donde sabemos que nuestros enfermos ven deteriorado importantemente este aspecto $^{13-16}$. No es inhabitual la necesidad de rehospitalización por deshidratación o desnutrición calórico proteica, problemas asociados a la herida operatoria, episodios de obstrucción intestinal y hernias de la pared abdominal. Todos estos problemas presentes en el mediano y largo plazo son evitados mediante la DSE propiciando una disminución en el gasto de recursos que claramente benefician al sistema de salud.

Probablemente una de las interrogantes e inquietudes más importantes en el largo plazo de quienes realizamos el tratamiento y seguimiento de pacientes con cáncer gástrico es el pronóstico oncológico. Diversos estudios han evaluado los resultados de la DSE en el largo plazo comparándolos con la cirugía resectiva. En general, la DSE ha sido asociada a una mayor tasa de recurrencia, pero con similar sobrevida global y específica por cáncer ${ }^{19-20}$. En nuestra serie no registramos recurrencias durante el seguimiento ni mortalidad por cáncer gástrico. La sobrevida global es de $98 \%$ similar a la reportada, tanto por nuestro grupo en casos de gastrectomías por $\mathrm{CGI}^{24}$ y por la literatura internacional ${ }^{26}$.

Una de las debilidades de nuestra serie es que aún constituye un seguimiento de solo 2 años por lo cual debemos estar atentos al seguimiento a más largo plazo. Sin embargo todo hace pensar que estos buenos resultados debieran mantenerse en el tiempo y que la DSE seguirá constituyendo nuestra alternativa de elección como grupo para el tratamiento del CGI.

El presente trabajo representa la mayor serie publicada a nivel nacional de DSE para el tratamiento del CGI. La morbilidad secundaria a la DSE es muy inferior a una gastrectomía y en un $86 \%$ de los casos es el único tratamiento curativo necesario. Estos resultados apoyan la amplia implementación de la técnica para el tratamiento del CGI.

\section{Responsabilidades éticas}

Protección de personas y animales. Los autores declaran que para esta investigación no se han realizado experimentos en seres humanos ni en animales.

Confidencialidad de los datos. Los autores declaran que en este artículo no aparecen datos de pacientes.

Conflictos de interés: no hay. 


\section{Bibliografía}

1. Ministerio de Salud. Guía Clínica AUGE Cáncer Gástrico. Santiago: Minsal, 2014.

2. Rugge M, Fassan M, Graham D. Epidemiology of Gastric Cancer. In: Strong V. (Ed). Gastric Cancer, Principles and practice. 1st Ed. Switzerland: Springer; 2015. p. 23.

3. Takahashi T, Saikawa Y, Kitagawa Y. Gastric cancer: current status of diagnosis and treatment. Cancers 2013;5:48-63.

4. Ajani JA, In H, Sano T, Gaspar L, Erasmus J, Tang L, et al. Stomach. In: Amin MB. (Ed). AJCC Cancer Staging Manual. 8th Ed. Chicago: AJCC; 2017. p. 203.

5. Katai H, Ishikawa T, Akazaxa K, Miyashiro I, Tsujitani S, Ono H, et al. Five-year survival analysis of surgically resected gastric cancer cases in Japan: a retrospective analysis of more than 100,000 patients from the nationwide registry of the Japanese Gastric Cancer Association (2001-2007). Gastric Cancer 2018; 21:144-54.

6. Kitano S, Shiraishi N, Uyama I, Sugihara $\mathrm{K}$, Tanigawa N, Japanese Laparoscopic Surgery Study Group (2007). A multicenter study on oncologic outcome of laparoscopic gastrectomy for early cancer in Japan. Ann Surg 2007;245:6872 .

7. Maehara Y, Orita H, Okuyama T, Moriguchi S, Tsujitani S, Korenaga D, et al. Predictors of lymph node metastasis in early gastric cancer. Br J Surg 1992;79:245.

8. Everett SM, Axon AT. Early gastric cancer in Europe. Gut 1997;41:142.

9. Yasuda K, Shiraishi N, Suematsu T, Yamaguchi K, Adachi Y, Kitano S. Rate of detection of lymph node metastasis is correlated with the depth of submucosal invasion in early stage gastric carcinoma. Cancer 1999;85:2119.

10. Okada K, Fujisaki J, Yoshida T, Ishikawa $\mathrm{H}$, Suganuma T, Kasuga A, et al. Longterm outcomes of endoscopic submucosal dissection for undifferentiated-type early gastric cancer. Endoscopy 2012;44:122-7.

11. Kim H, Hyung W, Cho G, Kim M, Han

S, Kim W, et al. Morbidity and mortality of laparoscopic gastrectomy versus open gastrectomy for gastric cancer: An interim report-A phase III multicenter, prospective, randomized trial (KLASS Trial). Ann Surg 2010;251:417-20.

12. Songun I, Putter H, Kranenbarg EM, Sasako M, van de Velde CJ. Surgical treatment of gastric cancer: 15-year follow-up results of the randomised nationwide Dutch D1D2 trial. Lancet Oncol 2010;11:439-49.

13. Kim SG, Ji SM, Lee NR, Park SH, You $\mathrm{JH}$, Choi IJ, et al. Quality of Life after Endoscopic Submucosal Dissection for Early Gastric Cancer: A Prospective Multicenter Cohort Study. Gut Liver 2016;11:87-92.

14. Choi JH, Kim ES, Lee YJ, Cho KB, Park KS, Jang BK, et al. Comparison of quality of life and worry of cancer recurrence between endoscopic and surgical treatment for early gastric cancer. Gastrointest Endosc 2015;82:299-307.

15. Hu J, Zhao Y, Ren M, Li Y, Lu X, Lu G, et al. The comparison between endoscopic submucosal dissection and surgery in gastric cancer: A systematic review and meta-analysis. Gastroenterol Res Pract 2018:4378945.

16. Kim YI, Kim YA, Kim CG, Ryu KW, Kim YW, Sim JA, et al. Serial intermediateterm quality of life comparison after endoscopic submucosal dissection versus surgery in early gastric cancer patients. Surg Endosc 2018;32:2114-22.

17. Gotoda T, Yanagisawa A, Sasako M, Ono H, Nakanishi Y, Shimoda T, et al. Incidence of lymph node metastasis from early gastric cancer: estimation with a large number of cases at two large centers. Gastric Cancer 2000;3:219-25.

18. Japanese Gastric Cancer Association. Japanese gastric cancer treatment guidelines 2014 (ver. 4). Gastric Cancer 2017;20:1-19.

19. Abdelfatah MM, Barakat M, Ahmad D, Ibrahim M, Ahmed Y, Kurdi Y, et al. Long-term outcomes of endoscopic submucosal dissection versus surgery in early gastric cancer: a systematic review and meta-analysis. Eur J Gastroenterol Hepatol 2019. doi: 10.1097/ MEG.0000000000001352.

20. Jeon HK, Kim GH, Lee BE, Park DY, Song GA, Kim DH, et al. Long-term outcome of endoscopic submucosal dissection is comparable to that of surgery for early gastric cancer: a propensitymatched analysis. Gastric Cancer 2018;21:133-43.

21. Donoso A, Sharp A, Parra-Blanco A, Roa JC, Bächler JP, Crovari F, et al. Disección submucosa endoscópica en cáncer gástrico incipiente: experiencia inicial en el Hospital Clínico de la Pontificia Universidad Católica de Chile. Rev Med Chile 2015;143:1277-85.

22. Galindo J, Rodríguez J, Norero E, Aguayo G, Pruyas M, Nilsen E, et al. Disección submucosa endoscópica en cáncer gástrico incipiente. Rev Chil Cir 2015;67:590-98.

23. Wang Z, Ma L, Zhang XM, Zhou ZX. Risk of lymph node metastases from early gastric cancer in relation to depth of invasion: experience in a single institution. Asian Pac J Cancer Prev 2014;15:5371-5.

24. Norero E, Vargas C, Achurra P, Ceroni M, Mejía R, Martínez C, et al. Survival and perioperative morbidity of totally laparoscopic versus open gastrectomy for early gastric cancer: Analysis from a single latin american centre. $\mathrm{ABCD}$ Arquivos Brasileiros de Cirurgía Digestiva 2019;32,e1413. doi: 10.1590/0102$672020180001 \mathrm{e} 1413$.

25. Oda I, Gotoda T, Hamanaka H, Eguchi T, Saito Y, Matsuda T, et al. Endoscopic submucosal dissection for early gastric cancer: technical feasibility, operation time and complications from a large consecutive cases. Dig Endosc 2005; 17:54-8.

26. Papenfuss WA, Kukar M, Oxenberg J, Attwood K, Nurkin S, Malhotra U, et al. Morbidity and mortality associated with gastrectomy for gastric cancer. Ann Surg Oncol 2014;21:3008-14. 